\title{
Estudio descriptivo del comienzo de la diabetes mellitus tipo 1 y sus familiares de primer grado
}

\author{
D. A. DE LUIS, M. ALONSO FERNÁNDEZ, M. GONZALEZ SAGRADO, R. ALLER, \\ O. IZAOLA, J. MARTÍN GIL, M. C. TERROBA, L. CUÉLLAR \\ Instituto de Endocrinología y Nutrición. Facultad de Medicina. Unidad de Apoyo a la \\ Investigación. Hospital Río Hortega. Universidad de Valladolid
}

\author{
DESCRIPTIVE STUDY OF ONSET DIABETES MELLITUS TYPE 1 \\ PATIENTS AND FIRST DEGREE FAMILIES
}

\section{RESUMEN}

Fundamento: Existe una escasez de estudios descriptivos en pacientes con debut de diabetes tipo 1. El objetivo de este trabajo ha sido analizar los datos clínicos y analíticos de los diabéticos tipo 1 en el momento del debut, así como en los familiares de primer grado.

Pacientes y método: Se estudiaron 47 diabéticos tipo 1 y 148 familiares de primer grado. Los datos fueron obtenidos a partir de los debuts de los diabéticos tipo 1 diagnosticados entre enero de 1999 y diciembre de 2002 en el Área de Valladolid Oeste. En todos los casos se recogieron datos antropométricos y se realizó una analítica básica, incluyendo autoanticuerpos.

Resultados: La edad media en el debut de la diabetes tipo 1 fue de $16,09(9,36)$ años, sin diferencias significativas entre varones (32 casos, $68 \%$ ) y mujeres (15 casos, $32 \%$ ), presentando una incidencia de 4,66 casos/100.000 habs./año. El valor medio de los autoanticuerpos antimicrosomales (antiTPO) y antidecarboxilasa del ácido glutámico (anti$\mathrm{GAD}$ ) presentó valores significativamente superiores en los casos que en los familiares, mientras que no se alcanzó significación estadística para los valores de anticuerpos antinsulina (AAI) y antitiroglobulina (antiTG). Los valores de autoanticuerpos tiroideos y pancreáticos no se correlacionaron entre sí, ni con la dosis de insulina utilizada al alta o la HbAlc del debut. Las dosis medias de insulina NPH y rápida al alta empleadas en los diabéticos fueron de $27,58(13,88) \mathrm{UI} / \mathrm{d}$ y $3,05(4,90) \mathrm{UI} / \mathrm{d}$ respectivamente.

Conclusiones: La edad de los debuts fue similar a otros estudios con mayor frecuencia de varones. Los valores de antiGAD y antiTPO fueron superiores en los casos que en los familiares, no existiendo diferencias en los valores de anti Tg y AAI. La autoinmunidad no se correlacionó con ningún parámetro de control glucémico.

PALABRAS CLAVE: Autoinmunidad. Debut. Diabetes mellitus tipo 1. Familiares de primer grado.

\section{ABSTRACT}

Background: Few information about descriptive studies of autoimmunity in diabetes mellitus type 1 onset patients and family exist. The aim of our study was to analyze clinical and biochemical parameters of onset diabetes mellitus type 1 patients and first degree families.

Patients and methods: Forty seven onset diabetes mellitus type 1 patients and 148 first degree families were studied, between January 1999 and December 2002, in the west area of Valladolid. In all cases and families an anthropometric study and biochemical determination with autoimmunity levels were performed.

Results: Average age in onset diabetes mellitus type 1 patients was 16.09 (9.36) years, without differences between males (32 cases, $68 \%$ ) and females (15 cases, 32\%), with an incidence of 4.66 cases $/ 100,000$ habs./year. Average values of peroxidase antibodies (antiPTO) and glutamic descarboxilase antibodies (antiGAD) were higher in diabetic patients than in families. No differences were detected in insulin antibodies (IAA) and tiroglobuline antibodies (antiTG). No correlation was detected between anti TPO and anti pancreas. Autoantibodies did not influence in glycemic control. Average dose of NPH insulin and regular insulin were 27.58 (13.88) UI/d y 3.05 (4.90) UI/d, respectively.

Conclusions: Average age of onset diabetes mellitus type 1 patients was similar than other studies, with a high frequency of males. VAlues of antiGAD y antiTPO were higher in diabetic patients than families. No correlation between autoimmunity and glycemic control was detected.

KEY WORDS: Autoimmunity. Debut. Diabetes mellitus type 1. First degree families.

de Luis DA, Alonso Fernández M, González Sagrado M, Aller R, Izaola O, Martín Gil J, Terroba MC, Cuéllar L. Estudio descriptivo del comienzo de la diabetes mellitus tipo 1 y sus familiares de primer grado. An Med Interna (Madrid) 2004; 21: 378-381.

\section{INTRODUCCIÓN}

En nuestro medio, la diabetes mellitus tipo 1 presenta una incidencia de 11 casos/100.000 habitantes (1) con tendencia a incrementarse, principalmente, en Europa y Asia (2). Recien- temente, se ha incorporado la determinación de autoanticuerpos como prueba de laboratorio útil en el diagnóstico de la enfermedad y en la detección precoz de los familiares de primer grado en riesgo de desarrollarla. En estos casos, por su mayor sensibilidad y especificidad, se suele emplear la deter-

Trabajo aceptado: 26 de marzo de 2004

Correspondencia: D. A de Luis. Instituto de Endocrinología y Nutrición. Universidad de Valladolid. C/ Perales, 16. 47130 Valladolid 
minación combinada de anticuerpos antiGAD y AAI (3). Por otra parte, resulta llamativa la escasez de estudios descriptivos de estos pacientes, así como su relación con la autoinmunidad tiroidea y la relación con parámetros de control.

El objetivo de este trabajo ha sido analizar los datos clínicos y analíticos de los diabéticos tipo 1 en el momento del debut, así como en los familiares de primer grado.

\section{PACIENTES Y MÉTODOS}

\section{PACIENTES}

El estudio ha incluido 47 pacientes diabéticos tipo 1 (pacientes con hiperglucemia, cetoacidosis y edad inferior a 35 años) diagnosticados entre enero de 1999 y diciembre de 2002, y a sus familiares de primer grado $(n=148)$. La captura de casos fue del $100 \%$, ya que el programa de debut de diabetes mellitus tipo 1 de nuestro área de salud conlleva a un ingreso hospitalario inicial. En el momento del debut se realizó una analítica completa en ambos grupos (hemograma, bioquímica con función renal y hepática, lipidograma, TSH, anticuerpos antitiroglobulina (antiTG), antiperoxidasas (antiTPO), anticuerpo antiinsulina (AAI) y anticuerpos antiglutámicodescarboxilasa (antiGAD). En los pacientes diabéticos también se realizó un equilibrio venoso y HbA1c. En todos los casos se midieron peso, talla e índice de masa corporal (IMC). En los pacientes diabéticos se recogieron los siguientes datos de la historia clínica: pérdida de peso en los meses previos, y dosis de insulina al alta.

\section{DETERMINACIONES BIOQUÍMICAS}

Las determinaciones de TSH se realizaron por inmunoanálisis (Architect, Abbott-Laboratories; Abbott Park, IL, EEUU), así como las de autoanticuerpos antiTG y antiTPO por ELISA (Gest; Menarini); se consideraron valores positivos de antiTG (>325 UI/ml) y de antiTPO (>50 UI/ml). Los anticuerpos antiGAD y AAI se determinaron por radioinmunoanálisis con valores de referencia: antiGAD negativo $<0.9 \mathrm{U} / \mathrm{ml}$ y AAI : 3,4$6,2 \%$ de fijación. Las variables bioquímicas (glucosa, urea, ácido úrico, creatinina, colesterol total y fracciones, proteínas totales, etc.) se determinaron en el autoanalizador Hitachi 917 (Roche Diagnostics, Mannheim, Germany) y la HbA1c mediante HPLC (HI-AutoA1c HA-8140, Arkay, Japan).

\section{ANÁLISIS ESTADÍSTICO}

Con los datos obtenidos se creó una base de datos empleando el paquete estadístico SPSS versión 11.0 para Windows, Inc; IL: EE.UU. Se realizó un análisis descriptivo de las variables cuantitativas determinando la media y la desviación estándar y las variables cualitativas se expresaron como frecuencias. Se analizó la distribución normal de las variables mediante la prueba de Kolmogorov-Smirnov. Se compararon los resultados de las variables cuantitativas la prueba de la t de Student, el test de la U de MannWhitney. Las variables cualitativas se evaluaron mediante el test de la Chi cuadrado (la prueba exacta de Fisher y la corrección de Yates). Se empleó el test de ANOVA asociado a la prueba de
Bonferroni para la comparación simultánea de medias. Se utilizaron los test de Pearson y Spearman, para evaluar la correlación entre las diferentes variables. Se consideraron valores estadísticamente significativos cuando $\mathrm{p}<0,05$.

\section{RESULTADOS}

La incidencia de diabetes mellitus tipo 1 en la población de Valladolid Oeste fue de 4.66 casos/100.000 habitantes. La distribución por sexos en el grupo de los casos fue de15 mujeres frente a 32 varones $(\mathrm{p}<0,05)$. En los familiares fue de 70 varones frente a 78 mujeres. La edad media de los pacientes fue de 16,09 $(9,36)$ años y 35,12 $(16,00)$ años en los familiares. La distribución por edad de los casos se aprecia en la figura 1. Se observó una mayor acumulación de casos para el rango de edad de 11 a 15 años (14 casos, $31,1 \%$ ) y el menor número de debuts se registró en mayores de 30 años $(3,6 \%)$. El mes con mayor número de debúts fue abril (8 casos, 17\%), seguido de marzo (6 casos, $12,7 \%)$. Los meses que presentaron un menor número de diagnósticos fueron octubre y diciembre (1 caso en cada uno de ellos, 2,13\%). El IMC fue significativamente menor en los casos en relación con los familiares $(19,23(3,92)$ vs $25,13(5,02)$; $\mathrm{p}<0,05)$. Este hecho se encuentra relacionado probablemente con la pérdida de peso propia del debut diabético $5,52(2,56) \mathrm{kg}$.

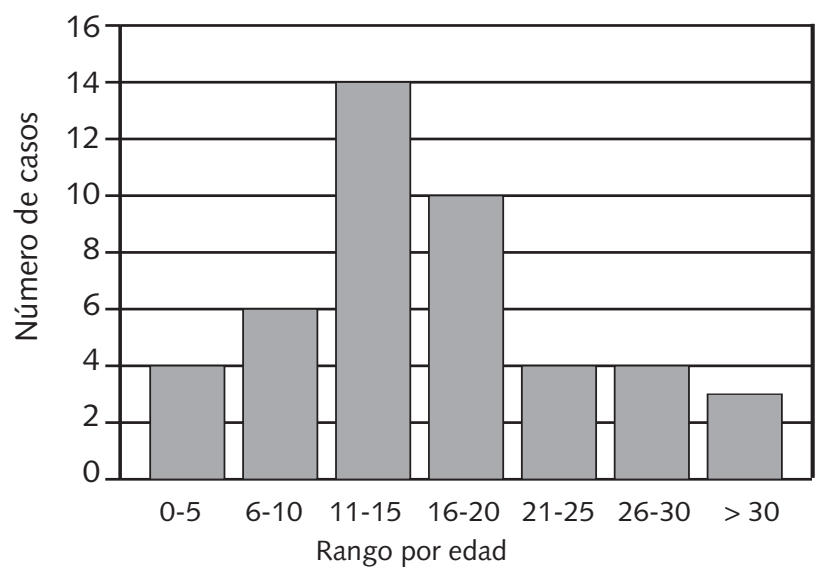

Fig. 1. Distribucion por edades de los debuts de diabetes tipo 1.

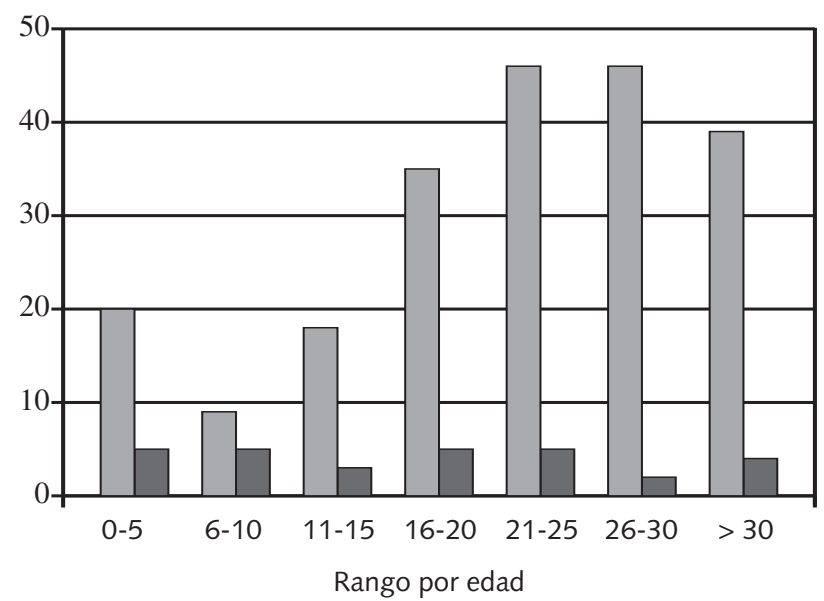

Fig. 2. Valores medios de antiGAD y AAl por edades. 
TABLA I

VALORES BIOQUÍMICOS DE CASOS Y FAMILIARES DE PRIMER GRADO

\begin{tabular}{lccc}
\hline & Casos & Familiares & Significación estadística \\
\hline Ǵlucosa $(\mathrm{mg} / \mathrm{dl})$ & $434,93(199,80)$ & $96,81(23,66)$ & 0,000 \\
Ácido úrico $(\mathrm{mg} / \mathrm{dl})$ & $9,36(35,53)$ & $4,64(1,21)$ & 0,000 \\
Fosfatasa alcalina $(\mathrm{mg} / \mathrm{dl})$ & $371,02(299,22)$ & $196,37(171,05)$ & 0,000 \\
TSH $(\mathrm{mUl} / \mathrm{ml})$ & $2,31(1,24)$ & $2,44(4,67)$ & N.S. \\
Colesterol total $(\mathrm{mg} / \mathrm{dl})$ & $172,79(50,65)$ & $185,82(44,23)$ & N.S. \\
Triglicéridos $(\mathrm{mg} / \mathrm{dl})$ & $102,90(96,49)$ & $78,47(41,24)$ & 0,048 \\
LDL colesterol $(\mathrm{mg} / \mathrm{dl})$ & $111,19(51,04)$ & $113,66(36,43)$ & 0,047 \\
HDL colesterol $(\mathrm{mg} / \mathrm{dl})$ & $46,86(10,56)$ & $58,88(14,79)$ & 0,021 \\
\hline
\end{tabular}

En la tabla I se recogen los resultados de las variables bioquímicas habituales. Los valores de HbA1c fueron de 11,39 $(1,71 \%)$, no existen diferencias significativas en cuanto al colesterol total y el índice de riesgo y si se aprecian éstas en los triglicéridos y las fracciones de colesterol.

Los valores de ACTPO $(265,8(792,83) \mathrm{UI} / \mathrm{ml}$ vs 140,94 $(523,117) \mathrm{UI} / \mathrm{ml} ; \mathrm{p}<0,05)$ y ACGAD $(26,69(37,83) \mathrm{UI} / \mathrm{ml} v s$ $0,91(4,45) \mathrm{UI} / \mathrm{ml} ; \mathrm{p}<0,05)$ fueron significativamente mayores en los pacientes diabéticos en relación a sus familiares de primer grado, presentando un mayor porcentaje de pacientes positivos que familiares ACTPO $(23,68$ vs $11,19 \%$; $\mathrm{p}<0,05)$ y $\operatorname{GAD}(65,78 v s 3,38 \% ; \mathrm{p}<0,05)$. Por el contrario, los ACTG y AAI no presentaron diferencias significativas, ni en términos absolutos ni en frecuencia de positividad.

En la figura 2 aparecen representados los valores medios de anticuerpos antiGAD y AAI en relación con la edad. En el caso de los antiGAD se observa una acumulación de los valores más altos en los pacientes con debut diabético de más de 15 años de edad, mientras que los anticuerpos AAI permanecen prácticamente constantes.

En cuanto al tratamiento, las dosis medias de insulina NPH e insulina rápida que precisaron los diabéticos en el momento del alta fueron de $27,58(13,88)$ y $3,05(4,90) \mathrm{UI} / \mathrm{d}$ respectivamente, con una media de 2,62 $(0,49)$ dosis subcutáneas al día.

En los análisis de correlación entre los diferentes autoanticuerpos no se detecto ninguna asociación. Tampoco se detectaron correlaciones entre los niveles de autoanticuerpos pancreáticos y el nivel de $\mathrm{HbAlc}$ en el momento del diagnóstico ni en los niveles de insulina necesarios para el control.

\section{DISCUSIÓN}

Nuestro trabajo muestra una población de debut de diabetes tipo 1 con una edad similar a la presente en otros trabajos, con unas tasas de autoanticuerpos (GAD) y (TPO) superior a los familiares de primer grado, sin mostrar ninguna correlación los autoanticuerpos con parámetros de control glucémico.

Aunque en la mayoría de los trabajos consultados no se recogen diferencias significativas en cuanto al sexo en los debuts diabéticos, algunos autores han señalado un discreto predominio en varones mayores de 15 años (5). En nuestros datos la distribución por sexos fue claramente desigual, con una proporción de varones del $68 \%$, siendo esta diferencia estadísticamente significativa, lo que concuerda con el estudio de Aguilera y cols. (1) donde también se evidenció un predominio claro de varones (62\% frente al $38 \%$ de mujeres). En cuanto a la distribución por edades, la mayor acumulación de casos se ha producido en el rango de edad de 11 a 15 años ( $31,1 \%$ de los casos), datos que son similares a los de otros estudios. En relación a los debuts en mayores de 15 años (46,6\% de los casos en nuestra muestra), existe mayor variabilidad en función de la población estudiada, de forma que Aguilera y cols. (1) obtuvieron resultados similares en este rango de edad (50\% de los casos) frente a otros estudios que observaron cifras más bajas (2).

Por otra parte, se han encontrado diferencias significativas en cuanto a la distribución de los debuts en función de la estación del año, diagnosticándose más casos en primavera y verano $(65,9 \%)$. Esta observación contrasta con la descrita en la literatura con un predominio de diagnósticos en otoño e invierno $(5,6)$.

En referencia a los datos antropométricos y la pérdida de peso en los meses previos al debút, en nuestro estudio se obtuvo un IMC de 19,23 $(3,92)$, inferior al publicado por Aguilera y cols. que fue de $21,3(3,7)$. Llama sin embargo la atención que la pérdida de peso fue mayor en su población que en la nuestra $(7,5(4,9)$ frente a 5,52 $(2,56))$. En el análisis por grupos se obtuvo una diferencia estadísticamente significativa al comparar el IMC de los casos, que fue de $19,23(3,92) \mathrm{kg} / \mathrm{m}^{2}$, frente al IMC de sus familiares de primer grado.

En relación con las variables bioquímicas básicas, las diferencias significativas observadas en las cifras de glucemia, triglicéridos y ácido úrico al comparar casos y familiares, se explican por la cetoacidosis propia del debut diabético. Las diferencias observadas en la fosfatasa alcalina están en relación con la menor edad de los casos frente a los familiares considerados en su conjunto y no se observan al hacer la comparación aislada con los hermanos.

En la bibliografía consultada se recoge que los antiGAD son positivos en el 70-80\% de los diabéticos de reciente diagnóstico y hasta en un $2 \%$ de individuos sanos $(1,4,7,8)$, siendo el mejor marcador de desarrollo de la enfermedad en familiares de primer grado y en la fase preclínica de la enfermedad (hasta diez años antes del inicio de los síntomas) $(9,10)$. Así mismo, son los que mejor se correlacionan con el daño en la célula $\beta$ y los más reproductibles y fiables. Paradójicamente, parece que los niveles más altos de los antiGAD en la fase preclínica se correlacionan con una progresión más lenta hacia la instauración de la enfermedad, fenómeno que se debe a que la activación preferente de la inmunidad humoral se hace a expensas de una menor activación de la celular. 
En cuanto a los anticuerpos antiinsulina, aparecen en diabéticos que no han recibido insulinoterapia y son positivos en el 30 a $50 \%$ de los casos en el debut $(1,4)$. Dado que existe una correlación inversa entre el título de los mismos y la edad al comienzo de la enfermedad, se suelen determinar en menores de 10 años. En la estudio de Aguilera y cols. (1), sobre una población de 86 pacientes se obtuvo una positividad para alguno de los autoanticuerpos analizados (antiGAD, antiinsulina y antiIA2) de un $80 \%$, mientras que la positividad para la combinación de antiGAD y antiinsulina se produjo en un $22 \%$ de los casos. En este trabajo se señala que sus porcentajes son inferiores a los observados por otros autores atribuyéndose esta diferencia a que excluyeron a los menores de 15 años, quienes clásicamente presentan niveles más altos de autoanticuerpos, exceptuando los antiGAD (11). En nuestra población, el $65,78 \%$ de los pacientes diabéticos presentaban antiGAD positivos en el momento de su debut, frente al 5,38\% del grupo de los familiares, siendo esta diferencia estadísticamente significativa. El análisis por grupos de edad, evidenció títulos más altos de antiGAD en los diabéticos mayores de 15 años al igual que en el estudio de Aguilera y cols. (1). Por otra parte, también se observaron diferencias en cuanto a la media observada de antiGAD con respecto a otras poblaciones, así en nuestro estudio se obtuvo una media de 26,69 $(37,83)$ muy superior al 7,11 $(13,4)$ de una muestra de diabéticos tipo 1 del Hospital Xeral de Vigo (8). Con respecto a los anticuerpos AAI, fueron positivos en el 14,70\% de los diabéticos tipo 1, valores inferiores a los recogidos en la bibliografía. Este dato puede estar en relación con la determinación de AAI en todos los debut, independientemente de su edad. El porcentaje de resultados positivos en los familiares de primer grado $(12,74 \%)$, no presentó diferencias estadísticamente significativas en relación a los casos, ni al hacer el análisis en función de grupos de edad, con valores discretamente más altos para el grupo de pacientes diabéticos de 6 a 10 años. Sin embargo, en ningún caso la media de anticuerpos AAI estuvo por encima de los valores de referencia.

En la bibliografía consultada se recoge una prevalencia de positividad para anticuerpos antitiroideos muy variable, oscilando entre el 3 y el $50 \%$ en función del país (14). En cuanto a los anticuerpos antimicrosomales y antitiroglobulina en el estudio de Aguilera y cols. (1) se obtuvo una positividad de 20,9 y $23,3 \%$ respectivamente. La positividad para antiTPO es el marcador más específico de trastorno tiroideo, de forma que en estos casos habría que determinar hormonas tiroideas anualmente para el despistaje de hipotiroidismo $(12,13)$. No obstante, las comparaciones para las determinaciones de autoanticuerpos (tanto antiGAD,AAI, antiTG y antiTPO) hay que hacerlas considerando varias limitaciones: en primer lugar en el estudio de Aguilera y cols. (1) no aparece reseñada la técnica de análisis ni los criterios de determinación de los puntos de corte, así como los valores de referencia empleados; de forma que no podemos asegurar que los resultados de confrontar ambos estudios sean exactos.

En conclusión, la edad de los debuts fue similar a otros estudios con mayor frecuencia de varones. Los valores de antiGAD y antiTPO fueron superiores en los casos que en los familiares, no existiendo diferencias en los valores de anti $\mathrm{Tg}$ y AAI. La autoinmunidad no se correlacionó con ningún parámetro de control glucémico.

\section{Bibliografía}

1. Aguilera A. Clinical, metabolic, immunologic and genotypic characteristics in non-pediatric patients with type $1 \mathrm{~A}$ diabetes mellitus tipo. Onset and short-term prognosis. Med Clin (Barc.) 2003; 120 (4): 121-4

2. P. Giralt Muiña. Incidencia en menores de 16 años y prevalencia de la diabetes mellitus tipo $1^{\mathrm{a}}$ en la provincia de Ciudad Real. Anales de Pediatria 2001; 55 (3): 213-218

3. Onkamo P. Worldwide increase in incidence of type 1 diabetes - the analysis of the data on published incidence trends. Diabetologia 1999; 42: 1395-1403

4. Recomendaciones de la Sociedad Española de Diabetes sobre la evaluación de riesgo de diabetes mellitus tipo 1.Endocrinología y nutrición 2002; 49 (6): 209-211

5. Alvarez Escolá C. Etiopatogenia de la diabetes mellitus. Medicine 2000; 8 (19): 991-1000.

6. Unger R. H.Diabetes Mellitus. Textbook of Endocrinology. Philadelphia: WB Saunders Company, 1998; 973-1060.

7. De Block CE, De Leeuw IH, Vertommen JJ, Rooman RP. Beta-cell, thyroid, gastric, adrenal and coeliac autoimmunity and HLA-DQ types in type 1 diabetes. Clin Exp Immunol 2001; 126: 184-186.

8. Alvarez E. Autoanticuerpos anti GAD y anti IA2 en Diabetes Mellitus tipo 1. Endocrinol Nutr 2003; 50 (Supl. 2): 22-87

9. Kulaeva TL. Genetic and immunologic aspects of type 1 diabetes mellitus. Usp Fiziol Nauk 2003; 34: 45-62

10. Krischer JP. Screening strategies for the identification of multiple antibody -positive relatives of individuals with type 1 diabetes. J Clin Endocrinol Metab 2003; 88:103-8

11. Torn C. Prognostic factors for the course of beta cell function in autoimmune diabetes. J Clin Endocrinol Metab 2000; 85: 4619-4623

12. Kordonouri O. Autoinmunidad tiroidea en niños y adolescentes con diabetes tipo 1. Diabetes Care 2003; 4 (2): 68-72.

13. Kordonouri O, Deiss D, Danne T, Dorow A, Bassin C, Gruters-Kieslich A. Predictivity of thyroid autoantibodies for the development of thyroid dsorders in children and adolescents with type 1 diabetes. Diabet Med 2002; 19; 518-521.

14. Chen BH, Chung SB, Chiag W, Chao MC. GAD 65 antibody prevalence and association with thyrid antibodies, HLA DR in Chinese children with type 1 diabetes mellitus. Diabetes Res Clin Pract 2001; $54 ; 27-32$. 